\title{
Novel therapeutic options for second-line therapy in metastatic renal cell carcinoma (Review)
}

\author{
CHRISTOPH-A. J. von KLOT ${ }^{1}$, AXEL S. MERSEBURGER ${ }^{2}$ and MARKUS A. KUCZYK ${ }^{1}$ \\ ${ }^{1}$ Department of Urology and Urological Oncology, Hannover University Medical School, D-30625 Hannover; \\ ${ }^{2}$ Department of Urology, Campus Lübeck University Hospital Schleswig-Holstein, D-23538 Lübeck, Germany
}

Received February 29, 2016; Accepted March 21, 2016

DOI: $10.3892 / \operatorname{mco} .2016 .856$

\begin{abstract}
Metastatic renal cell carcinoma (mRCC) has gained a variety of therapeutic options since the introduction of targeted therapy, starting in 2007. The basic molecular mechanisms included predominantly the targeting of vascular endothelial growth factor or the inhibition of the mammalian target of rapamycin. Recently, results from two randomized controlled trials, the CheckMate-25 and the METEOR trial, regarding therapy for $\mathrm{RCC}$ in the second-line setting have been published. In the present review, the current status of second-line therapy in $\mathrm{mRCC}$ is discussed, together with results from the two newly introduced substances, nivolumab and cabozantinib.
\end{abstract}

\section{Contents}

1. Introduction

2. Randomized controlled trials on second-line therapy for advanced renal cell cancer

3. Nivolumab and cabozantinib as second-line therapy options

4. Nivolumab

5. Cabozantinib

6. Topics of discussion

7. Conclusion

\section{Introduction}

Renal cell carcinoma (RCC) comprises $2-3 \%$ of all malignancies (1). In 2012, there were 34,700 kidney cancer-associated

Correspondence to: Dr Christoph-A J. von Klot, Department of Urology and Urological Oncology, Hannover University Medical School, 1 Carl Neuberg Street, D-30625 Hannover, Germany

E-mail: Klot.Christoph@mh-hannover.de

Key words: renal cell carcinoma, vascular endothelial growth factor, rapamycin, nivolumab, cabozantinib mortalities in the European Union (2). With the broad introduction of imaging modalities to detect early RCC, a trend towards newly diagnosed early stage RCC has ensued (3). Nonetheless, $\sim 30 \%$ of patients initially present with already metastasized disease (4), and approximately every third patient with localized RCC will develop recurrent and progressive cancer (5). Cure rates for patients with low risk RCC are excellent; however, 5-year progression-free survival (PFS) rates quickly fall with higher initial stages of RCC, adding to the pool of patients heading towards metastatic disease (6).

It was less than a decade ago that metastatic (m)RCC was considered to be rather unaffected by any type of systemically applied substance. Interleukin-2 and interferon were able to achieve median overall survival (OS) rates of $\sim 12$ months. High-dose interleukin-2 was the only FDA (United States Food and Drug Administration) approved substance for the treatment of RCC up until 2007 (7,8). With the advent of targeted therapy, median PFS almost doubled from 5 to 11 months, and median OS increased to almost 30 months (9-11). A variety of randomized controlled trials (RCTs) were assessing patients according to pretreatment with cytokines. The use of cytokines, however, does not reflect the current standard of treatment in the Western world today, and will most likely not be subjected to consideration when assessing second-line sequencing. At present, patients have a wide array of therapeutic options, and they will inevitably enter a sequence of targeted therapies over the course of their disease. After failure of first-line targeted therapy, there are various options for second-line treatment. The current available options for clear cell RCC (ccRCC) second-line treatment include the multitargeted tyrosine kinase inhibitors, axitinib (12), sorafenib (13) and pazopanib (14), as well as the mammalian target of rapamycin (mTOR) inhibitor, everolimus (15).

\section{Randomized controlled trials on second-line therapy for advanced renal cell cancer}

The current recommendations allow for several substances to be selected from, i.e. there are no firm recommendations as to the optimal sequence of targeted therapy (16). The following discussion of sequencing options, and the discussed RCTs in the second-line setting, all have a highly heterogeneous patient population with differing first-line treatment regimens, thus making the interpretation of the results all the more 
challenging when comparing decision making in the first-line setting. These differences in study populations have to be considered when discussing the variety of previous studies that were addressing the question of second-line therapy in mRCC and advanced RCC: Second-line therapy following progression on sunitinib was tested for temsirolimus versus sorafenib in the Investigating Torisel As SecondLine Therapy (INTORSECT) RCT. Median OS was longer in the sorafenib arm when compared with temsirolimus (16.6 vs. 12.3 months). This finding revealed that tyrosine kinase inhibition in the second-line setting was superior to mTOR inhibition with temsirolimus. The INTORSECT trial allowed for any histology, not only ccRCC (17).

Second-line axitinib following sunitinib produced a median OS rate of 20.1 months when compared with sorafenib, with 19.2 months in the advanced RCC (AXIS) trial (12). The AXIS trial was able to reveal an interesting association between exposure time to first-line sunitinib with PFS in the second-line sequence with axitinib. Although OS did not differ between the two substances, PFS was longer in the axitinib (8.3 vs. 5.7 months) arm. Axitinib is currently established in all risk groups as a second-line therapy option for mRCC.

The SWITCH-I study investigated the sequence of sunitinib following sorafenib vs. sorafenib following sunitinib. There were, however, no significant differences observed with regard to OS or PFS.

As an mTOR complex inhibitor, everolimus does not predominantly block growth factor receptors, but inhibits the regulatory transcription factors of pathways that regulate cell proliferation, angiogenesis and cell survival. The renal cell cancer treatment with oral RAD001 given daily (RECORD)-1 trial compared everolimus with a placebo in the second-line setting, and revealed a similar median OS of 14.8 vs. 14.4 months (15). In this study, patients with mRCC were included who experienced a progression of the disease under ongoing treatment with sunitinib and/or sorafenib. The median PFS survival was 4.0 vs. 1.9 months in favor of everolimus, however, as in numerous trials, the setting cannot be regarded as truly second-line therapy, since patients in this RCT were allowed to have had more than one previous treatment for mRCC prior to study inclusion. The multicenter randomized phase II trial RECORD-3 included 471 patients with good and intermediate prognosis, and assessed the sequence of everolimus/sunitinib versus sunitinib/everolimus in a crossover design (18). The primary endpoint of non-inferiority of first-line everolimus in this setting was not met. Median PFS for sunitinib following everolimus was 21.1 months compared with 25.8 months for the sequence with initial sunitinib [hazard ratio $(\mathrm{HR})=1.3,95 \%$ confidence interval (CI): 0.9-1.7]. OS was also longer in the sequence with sunitinib administered in first-line therapy (32 vs. 22.4 months; $\mathrm{HR}=1.2$, 95\% CI: 0.9-1.6).

A systematic review of the above-mentioned trials is shown in Table I.

\section{Nivolumab and cabozantinib as second-line therapy options}

With the introduction of targeted therapy in 2007, a variety of substances were introduced in rapid succession. These newly introduced substances comprised a vast variety of different modes of action. The novel molecular therapeutic mechanisms included the targeting of vascular endothelial growth factor (VEGF) or the inhibition of mTOR (19). Everolimus belongs to the substance family of mTOR inhibitors. It is currently recommended as treatment option for mRCC as second-line therapy (19). This means that it will currently be administered following targeted therapy with substances such as Sutent ${ }^{\circledR}$ (sunitinib), pazopanib and bevacizumab. Everolimus was tested in the sequence following first-line therapy against nivolumab in the CheckMate-25 trial (20), as well as against cabozantinib in the recently published METEOR trial (21).

\section{Nivolumab}

The results of a phase III trial of nivolumab $(3 \mathrm{mg} / \mathrm{kg}$ body weight) versus everolimus (10 mg daily) in 821 patients with progressing advanced RCC was introduced at the ESMO 2015 meeting in Vienna and simultaneously published in the New England Journal of Medicine (20). Nivolumab is a human immunoglobulin (Ig)G4 monoclonal antibody that blocks the programmed cell death receptor (PD-1), which is expressed on activated T-cells. It also blocks PD-1 ligand 1 (PD-L1), which is expressed on the tumor cells. This checkpoint inhibition results in the activation of T-cells that are normally deactivated by PD1-1 due to binding with PD-L1 $(22,23)$. Only patients with clear cell histology were included. Patients had received $\geq 1$, but not $>3$, regimens of therapy, including targeted and cytokine therapy, prior to the study. However, more than two-thirds of all patients had only received one previous regimen prior to the study. Patients were not allowed to have had previous treatment with everolimus or any other mTOR inhibitor. Patients were stratified according to the Memorial Sloan Kettering Cancer Center (MSKCC) prognostic risk classification system, taking into account performance status, hemoglobin and serum calcium levels (24). The primary endpoint was OS. Notably, the expression of PD-L1 on the tumor membrane was also evaluated compared with the response to therapy. Quality of life was assessed with the Functional Assessment of Cancer Therapy-Kidney Symptom Index-Disease-Related Symptoms score (FKSI-DRS) (25). The study was stopped prematurely in July 2015, when the independent data monitoring committee concluded that a significant benefit with regard to OS was observed in the nivolumab arm. Median OS was significantly higher in the nivolumab group (25.0 vs. 19.6 months). The HR for mortality was 0.73 (98.5\% CI: $0.57-0.93 ; \mathrm{P}=0.002)$ in favor of nivolumab. The survival benefit was observed in all subgroups. PFS did not differ significantly between nivolumab and everolimus (4.6 vs. 4.4 months; $\mathrm{P}=0.11$ ). The two therapeutic regimes were well tolerated, with $19 \%$ grade 3 or 4 adverse events in the nivolumab arm, and $37 \%$ grade 3 or 4 adverse events in the everolimus arm. The most common adverse event for nivolumab was fatigue, whereas anemia was the most common event with everolimus. Although no patient died in the nivolumab arm, two mortalities occurred with everolimus, which were attributable to bowel ischemia and septic shock. The superiority of nivolumab with regard to OS was demonstrated across a variety of baseline factors, such as the 
Table I. Selection of RCTs with evaluation of a second-line targeted therapy regimen for renal cell carcinoma.

\begin{tabular}{|c|c|c|c|c|c|}
\hline Trial & Therapy & Prior regimens & PFS, month & OS, month & Refs. \\
\hline AXIS & Axitinib vs. sorafenib & $\begin{array}{l}\text { RCC, one prior therapy } \\
\text { including cytokines }\end{array}$ & 8.3 vs. 5.7 & 20.1 vs. 19.2 & $(12)$ \\
\hline INTORSECT & Sorafenib vs. temsirolimus & $\begin{array}{l}\text { Any RCC histology, second } \\
\text { line following sunitinib }\end{array}$ & 3.9 vs. 4.3 & 16.6 vs. 12.3 & $(17)$ \\
\hline SWITCH-I & $\begin{array}{l}\text { Sunitinib following sorafenib } \\
\text { vs. sorafenib following } \\
\text { sunitinib }\end{array}$ & None & 12.5 vs. 14.9 & 31.5 vs. 30.2 & $(39)$ \\
\hline RECORD-1 & Everolimus vs. placebo & $\begin{array}{l}\text { ccRCC, progression following } \\
\text { sunitinib and/or sorafenib }\end{array}$ & 4.9 vs. 1.9 & 14.8 vs. 14.4 & $(40)$ \\
\hline RECORD-3 & $\begin{array}{l}\text { Sunitinib/everolimus } \\
\text { vs. everolimus/sunitinib }\end{array}$ & $\begin{array}{l}\text { Good and intermediate } \\
\text { prognosis RCC }\end{array}$ & 25.8 vs. 21.1 & 32 vs. 22.4 & $(18)$ \\
\hline METEOR & Cabozantinib vs. everolimus & $\begin{array}{l}\mathrm{ccRCC}, \geq 1 \text { prior } \\
\text { targeted therapy }\end{array}$ & 7.4 vs. 3.8 & Not reached & $(21)$ \\
\hline CheckMate-025 & Nivolumab vs. everolimus & $\begin{array}{l}\text { ccRCC, } \geq 1 \text { prior } \\
\text { targeted therapy }\end{array}$ & 4.6 vs. 4.4 & 25.0 vs. 19.6 & $(20)$ \\
\hline
\end{tabular}

(cc)RCC, (clear cell) renal cell carcinoma; INTORSECT, Investigating Torisel As Second-Line Therapy; RECORD, Renal cell cancer treatment with Oral RAD001 given daily; RCT, randomized controlled trial; PFS, progression-free survival; OS, overall survival.

Karnofsky index and number of prior therapies, as recently demonstrated at the Genitourinary Cancers Symposium 2016 (26).

These results are promising, but one must bear in mind that, by far, the majority of patients did not respond to therapy at all: The overall objective response rate for nivolumab was $25 \%$, and for everolimus, $5 \%$ (odds ratio $=5.98,95 \%$ CI: 3.68-9.72; $\mathrm{P}<0.001$ ), signifying that four out of five patients did not even respond to therapy in the second-line setting. The median duration of the response was 12 months, with a maximum of achievable response duration of 27.6 and 22.2 months for nivolumab and everolimus, respectively. Another observation, crucial for monitoring patients, is the fact that median time to response was 3.5 months (range, 1.4-24.8 months) in the nivolumab arm, and 3.7 months in the everolimus group (range, 1.5-11.2 months). This is an important issue when taking into account that the median treatment duration was only 5.5 months for nivolumab.

In the phase II trial assessment, the efficacy of nivolumab in $\mathrm{mRCC}$ PD-L1 expression was quantifiable in $64 \%$ of the patients. Approximately one-third of these patients exhibited a PD-L1 expression level of $\geq 5 \%$. A PD-L1 expression level $\geq 5 \%$ was markedly associated with longer PFS and OS. Similarly, other studies were able to show an association between co-stimulatory markers for T-cell function with renal cell cancer aggressiveness: TB7-H1, a glycoprotein which has been shown to increase apoptosis of T-cells and elevate the expression of $\mathrm{TB} 7-\mathrm{H} 1$, is a marker for more aggressive RCC variants (27). Therefore, evaluation of PD-L1 expression in correlation with treatment response and survival was of special interest in subsequent trials of nivolumab for RCC.

In the current phase III study by Motzer et al (20), 821 patients were tested, and $92 \%$ of the patients exhibited quantifiable PD-L1 expression. Patients were dichotomized at expression levels of $1 \%$, and also at $5 \%$. OS for patients with a PD-L1 expression level $\geq 1 \%$ was 21.8 months for nivolumab, and 18.8 months for everolimus. OS for patients with $<1 \%$ PD-L1 expression was 27.4 months in the nivolumab group, and 21.2 months in the everolimus group. Similar results were observed when dichotomizing expression of PD-L1 at the $5 \%$ level. These results demonstrated that nivolumab did not exert a greater effect on patients with a higher expression of PD-L1. Irrespective of PD-L1 expression, nivolumab was associated with superior OS compared with everolimus. The discrepancy between the current CheckMate-025 trial and previously conducted similar studies cannot be fully accounted for at present. One hypothetical explanation could be that expression levels of PD-L1 are subjected to change during the course of disease progression, and may even change under immune therapy. Similar changes have previously been reported for non-small cell lung cancer by immunohistochemical analysis (28).

\section{Cabozantinib}

Cabozantinib is an orally administered tyrosine kinase inhibitor (TKI). It targets the VEGF receptor, together with the tyrosine kinases MET and AXL, which exert a role in the development of mRCC. As such, MET and AXL are both up-regulated in RCC. Elevated expression levels of MET and AXL are linked with a poorer prognosis $(29,30)$. Cabozantinib has already been approved for patients with medullary thyroid cancer (31). Patients pretreated with VEGF TKIs exhibited c-MET upregulation, which logically leads to a strategy of therapeutic MET inhibition via cabozantinib. The METEOR study addressed the question of therapeutic efficacy for everolimus versus cabozantinib in the second-line treatment of mRCC (21) in a 1:1 (cabozantinib, $60 \mathrm{mg} /$ day: everolimus, $10 \mathrm{mg}$ /day) RCT with an 
intention-to-treat setting. The primary endpoint in this study was PFS. The secondary endpoints comprised OS and the objective response rate. All 658 patients had ccRCC, and must have progressed while under treatment with at least one prior VEGF receptor inhibitor. Patients were only eligible with a Karnofsky performance status of $\geq 70 \%$, although there was no limit on the number of previous anti-tumoral regimens; only patients with prior treatment with an mTOR inhibitor were excluded. The majority of patients received one prior therapy with sunitinib prior to entering the study. Patients were stratified by risk group according to the MSKCC criteria (24), and also according to the number of the previous targeted therapies. Radiographic response rates were higher in the cabozantinib arm (21 vs. $5 \%)$. The median PFS was 7.4 and 3.8 months for cabozantinib and everolimus, respectively. The HR for progression of disease or mortality was 0.58 (95\% CI: $0.45-0.75 ; \mathrm{P}<0.001)$ in favor for cabozantinib. The PFS advantage for cabozantinib was observed in all subgroups specified by the RCC prognostic risk (low, intermediate and high), and subgroups defined by the number of prior targeted therapy regimes. The effect was greatest for the subgroup with sunitinib as sole prior therapy (9.1 vs. 3.7 months for cabozantinib and everolimus, respectively). The most prominent cause for discontinuation of the assigned study treatment was radiographic progression. OS was assessed at the pre-specified interim analysis stage, which revealed a trend towards longer OS for cabozantinib (HR for mortality $=0.67$, 95\% CI: 0 0.51-0.89; $\mathrm{P}=0.005$ ); however, the required $\mathrm{P}$-value of $\leq 0.0019$ for statistical significance was not reached, and assumptions on OS are still required to be made with caution due to the immature nature of the data. During treatment, toxicity and adverse events differed between cabozantinib and everolimus. Dose reduction was more often necessary in the cabozantinib arm when compared with everolimus (60 vs. $25 \%$ of cases). Adverse events grades 3 or 4 occurred in $68 \%$ of cases with cabozantinib, and in $58 \%$ for everolimus.

\section{Topics of discussion}

The presented data suggest second-line therapy with PD-L1 inhibition or VEGF receptor inhibitors, particularly as presented with regard to nivolumab and cabozantinib, which may have greater efficacy following first-line therapy compared with mTOR inhibition. The data presented in this review, particularly for the METEOR trial, do not describe a unique sequence of two substances, since various first-line regimens were allowed, including multiple substances prior to cabozantinib. Currently, there is growing evidence supporting the idea of VEGF receptor inhibition in the second-line setting, which is supposedly superior to everolimus, i.e. mTOR inhibition. Differing sequences in phase III trials for second-line therapy are, however, a problem. The AXIS trial allowed for different prior therapies as well, and even cytokine treatment was allowed as a first regimen. Analyzing previous studies with regard to results derived from administering nivolumab or cabozantinib is difficult, since differences in patient selection and trial design do not allow for a direct comparison. It will be interesting to see whether these substances, with their different modes of action, will lead to a further improved response if administered simultaneously.

The combination of these newly introduced therapeutic approaches (cabozantinib in combination with nivolumab) is currently being researched in a randomized trial setting together or in comparison with ipilimumab. The trial will also address other urological tumors, aside from RCC (ClinicalTrials.gov identifier NCT02496208).

The CheckMate-025 and the METEOR trial had ccRCC as the predominant histopathological finding. The two trials did not favor everolimus as a second-line treatment. The question remains whether this conclusion can be drawn for non-ccRCC. An interesting consideration regarding everolimus, in particular, emerged from the ASPEN trial that was recently presented at the American Society of Clinical Oncology (ASCO) Convention 2015, held in Chicago. The results demonstrated that a subset of patients with non-ccRCC may benefit from everolimus, in particular. The study was, however, investigating the first-line setting. The ASPEN trial included 108 patients who were subjected to systemic therapy with sutent or everolimus. The trial revealed overall superior results for sutent; notably, the subset of patients with chromophobe histology had a PFS of 11.5 vs. 5.6 months in favor of everolimus (32). It may not be valid to draw premature conclusions from these results, since the absolute number of patients responding to everolimus was rather low, comprising only $15 \%$ of the already small study population. Therefore, the results may not be enough to justify a paradigm shift in current therapeutic concepts or negate results from the CheckMate-025 or the METEOR trial.

The question remains why cabozantinib did, in fact, show superior PFS in the METEOR trial. Results from the AXIS trial showed a median PFS of 4.8 months for the subgroup that had been previously treated with sunitinib prior to axitinib. It may be due to the fact that cabozantinib not only targets the VEGF receptor, but also acts on MET and AXL unrelated to TKIs $(29,30)$, which leads to longer PFS. The benefit of a novel mode of action also pertains to nivolumab. The two newly introduced substances as a second-line option for therapy may therefore be of special interest in patients with RCC that is primarily refractory to initial anti-VEGF therapy. It is known that patients which show 'progressive disease' as best response after initiation of the targeted therapy have dismal OS following second-line treatment, as was previously shown for 1,056 patients by Heng et al (33): Patients who initially failed VEGF inhibitor therapy only had a PFS of 2.4 months during subsequent therapy.

The significance of PFS in the METEOR and in the CheckMate-25 trial is also a topic of discussion. PFS cannot unconditionally be regarded as a surrogate parameter for OS. The INTORSECT trial, for example, did not reveal any significant difference in PFS, while showing a clear advantage in OS in favor for sorafenib in comparison with second-line therapy with temsirolimus. The difference in PFS between nivolumab and everolimus in the CheckMate-25 trial did not reach statistical significance, with 4.6 months for nivolumab vs. 4.4 months for everolimus, whereas OS was significantly superior in the nivolumab arm, as mentioned above. The discrepancy between PFS and OS could possibly be explained by a potential delayed benefit with nivolumab. 
A subgroup of patients who did not have disease progression or died at 6 months exhibited a marked difference in PFS between nivolumab and everolimus (15.6 vs. 11.7 months; $\mathrm{HR}=0.64$, 95\% CI: 0.47-0.88). One possible explanation for initial poor PFS, or a delayed effect of therapy, may be the known 'pseudo-progression' that had previously been reported for ipilimumab in patients with advanced melanoma. The phenomenon describes a radiographic growth of tumor lesions during the initial phase of therapy that is followed by subsequent regression. This phenomenon had previously been observed in patients with advanced melanoma treated with the monoclonal antibody against cytotoxic T-lymphocyte-associated protein 4, ipilimumab (34). The initial radiographic progression has been attributed to a delayed immune response or edema associated with therapy. In an attempt to design response criteria for patients with solid tumors under novel immune therapy, several patterns of response have been identified: i) initial shrinkage; ii) stable disease; iii) response following initial growth of the tumor; and iv) response in the presence of new lesions. It has been shown that all these patterns were associated with a favorable effect on OS (35). Therefore, the response evaluation criteria in solid tumors, which have been designed to assess effects of cytotoxic substances (36), may not provide an optimal assessment of treatment response under checkpoint inhibition. These findings may be one reason for discrepancies in PFS evaluation and OS.

Adverse events in the METEOR trial, as well as in the CheckMate-25 trial, did not show any surprising results: The percentages of grade 3 or 4 adverse reactions for everolimus were $37 \%$ in the CheckMate-25 trial and 58\% in the METEOR trial. In contrast, nivolumab revealed only $19 \%$ of grade 3 or 4 adverse reactions in the CheckMate-25 trial, whereas cabozantinib was associated with $68 \%$ of serious adverse reactions in the METEOR trial. The most prevalent adverse reaction for nivolumab was fatigue. Although nivolumab was well tolerated overall, one must bear in mind that adverse reactions in the form of inflammatory reactions may arise that are new to the clinician's eye, but which nonetheless can be serious and must be considered when administering novel checkpoint inhibition therapy. These adverse events may include skin rashes, hepatitis, pneumonitis and other less common inflammatory events. Adverse reactions, including, for instance, hypophysitis, may feature non-specific symptoms, which must also be considered when applying checkpoint inhibition therapy $(20,21,37,38)$.

\section{Conclusion}

The newly introduced substances for second-line therapy, nivolumab and cabozantinib, in advanced and mRCC have yielded promising results with regard to OS and PFS. A clear benefit regarding OS for cabozantinib remains to be conclusively demonstrated. Immune therapy, most likely, will improve therapeutic options for patients with advanced RCC in the future. With the introduction of novel mechanisms of action, physicians must be aware of differences in evaluating radiographic responses, as well as remain vigilant for a set of as-yet-unfamiliar and rare adverse reactions to checkpoint inhibitory therapy.

\section{References}

1. European Network of Cancer Registries (ENCR): Eurocim. Version 4.0. European incidence database v2.3, 730 entity dictionary. ENCR: Lyon, 2001.

2. Ferlay J, Steliarova-Foucher E, Lortet-Tieulent J, Rosso S, Coebergh JWW, Comber H, Forman D and Bray F: Cancer incidence and mortality patterns in Europe: Estimates for 40 countries in 2012. Eur J Cancer 49: 1374-1403, 2013.

3. Katz DL, Zheng T, Holford TR and Flannery J: Time trends in the incidence of renal carcinoma: Analysis of connecticut tumor registry data, 1935-1989. Int J Cancer 58: 57-63, 1994.

4. Fisher R, Gore M and Larkin J: Current and future systemic treatments for renal cell carcinoma. Semin Cancer Biol 23: 38-45, 2013.

5. Kroeger N, Choueiri TK, Lee J-L, Bjarnason GA, Knox JJ, MacKenzie MJ, Wood L, Srinivas S, Vaishamayan UN, Rha SY, et al: Survival outcome and treatment response of patients with late relapse from renal cell carcinoma in the era of targeted therapy. Eur Urol 65: 1086-1092, 2014.

6. Keegan KA, Schupp CW, Chamie K, Hellenthal NJ, Evans CP and Koppie TM: Histopathology of surgically treated renal cell carcinoma: Survival differences by subtype and stage. J Urol 188: 391-397, 2012.

7. Franklin JR, Figlin R, Rauch J, Gitlitz B and Belldegrun A: Cytoreductive surgery in the management of metastatic renal cell carcinoma: The UCLA experience. Semin Urol Oncol 14: 230-236, 1996.

8. Sarna G, Figlin R and de Kernion J: Interferon in renal cell carcinoma. The UCLA experience. Cancer 59 (Suppl 3): 610-612, 1987.

9. Motzer RJ, Hutson TE, Tomczak P, Michaelson MD, Bukowski RM, Oudard S, Negrier S, Szczylik C, Pili R, Bjarnason GA, et al: Overall survival and updated results for sunitinib compared with interferon alfa in patients with metastatic renal cell carcinoma. J Clin Oncol 27: 3584-3590, 2009.

10. Motzer RJ, Hutson TE, Tomczak P, Michaelson MD, Bukowski RM, Rixe O, Oudard S, Negrier S, Szczylik C, Kim ST, et al: Sunitinib versus interferon alfa in metastatic renal-cell carcinoma. N Engl J Med 356: 115-124, 2007.

11. Motzer RJ,Hutson TE,CellaD, Reeves J,Hawkins R, Guo J,Nathan P, Staehler M, de Souza P, Merchan JR, et al: Pazopanib versus sunitinib in metastatic renal-cell carcinoma. N Engl J Med 369: 722-731, 2013.

12. Motzer RJ, Escudier B, Tomczak P, Hutson TE, Michaelson MD, Negrier S, Oudard S, Gore ME, Tarazi J, Hariharan S, et al: Axitinib versus sorafenib as second-line treatment for advanced renal cell carcinoma: Overall survival analysis and updated results from a randomised phase 3 trial. Lancet Oncol 14: 552-562, 2013.

13. Escudier B, Eisen T, Stadler WM, Szczylik C, Oudard S, Siebels M, Negrier S, Chevreau C, Solska E, Desai AA, et al; TARGET Study Group: Sorafenib in advanced clear-cell renal-cell carcinoma. N Engl J Med 356: 125-134, 2007.

14. Sternberg CN, Davis ID, Mardiak J, Szczylik C, Lee E, Wagstaff J, Barrios CH, Salman P, Gladkov OA, Kavina A, et al: Pazopanib in locally advanced or metastatic renal cell carcinoma: Results of a randomized phase III trial. J Clin Oncol 28: 1061-1068, 2010.

15. Motzer RJ, Escudier B, Oudard S, Hutson TE, Porta C, Bracarda S, Grünwald V, Thompson JA, Figlin RA, Hollaender N, et al; RECORD-1 Study Group: Efficacy of everolimus in advanced renal cell carcinoma: A double-blind, randomised, placebo-controlled phase III trial. Lancet 372: 449-456, 2008.

16. Ljungberg B (chair), Bensalah K, Bex A (vice-chair), Canfield S, Dabestani S, Hofmann F, Hora M, Kuczyk MA, Lam T, Marconi L, et al: Guidelines on Renal Cell Carcinoma. European Association of Urology, 2015.

17. Hutson TE, Escudier B, Esteban E, Bjarnason GA, Lim HY, Pittman KB, Senico P, Niethammer A, Lu DR, Hariharan S, et al: Randomized phase III trial of temsirolimus versus sorafenib as second-line therapy after sunitinib in patients with metastatic renal cell carcinoma. J Clin Oncol 32: 760-767, 2014.

18. Motzer RJ, Barrios CH, Kim TM, Falcon S, Cosgriff T, Harker WG, Srimuninnimit V, Pittman K, Sabbatini R, Rha SY, et al: Phase II randomized trial comparing sequential first-line everolimus and second-line sunitinib versus first-line sunitinib and second-line everolimus in patients with metastatic renal cell carcinoma. J Clin Oncol 32: 2765-2772, 2014. 
19. Escudier B, Porta C, Schmidinger M, Algaba F, Patard JJ, Khoo V, Eisen T and Horwich A; ESMO Guidelines Working Group: Renal cell carcinoma: ESMO Clinical Practice Guidelines for diagnosis, treatment and follow-up. Ann Oncol 25 (Suppl 3): iii49-iii56, 2014.

20. Motzer RJ, Escudier B, McDermott DF, George S, Hammers HJ, Srinivas S, Tykodi SS, Sosman JA, Procopio G, Plimack ER, et al: CheckMate 025 Investigators: Nivolumab versus everolimus in advanced renal-cell carcinoma. N Engl J Med 373: 1803-1813, 2015.

21. Choueiri TK, Escudier B, Powles T, Mainwaring PN, Rini BI, Donskov F, Hammers H, Hutson TE, Lee J-L, Peltola K, et al; METEOR Investigators: Cabozantinib versus everolimus in advanced renal-cell carcinoma. N Engl J Med 373: 1814-1823, 2015

22. Hamid O and Carvajal RD: Anti-programmed death-1 and anti-programmed death-ligand 1 antibodies in cancer therapy. Expert Opin Biol Ther 13: 847-861, 2013.

23. Hamanishi J, Mandai M, Iwasaki M, Okazaki T, Tanaka Y, Yamaguchi K, Higuchi T, Yagi H, Takakura K, Minato N, et al: Programmed cell death 1 ligand 1 and tumor-infiltrating CD8+ T lymphocytes are prognostic factors of human ovarian cancer. Proc Natl Acad Sci USA 104: 3360-3365, 2007.

24. Motzer RJ, Bacik J, Schwartz LH, Reuter V, Russo P, Marion S and Mazumdar M: Prognostic factors for survival in previously treated patients with metastatic renal cell carcinoma. J Clin Oncol 22: 454-463, 2004

25. Cella D, Yount S, Brucker PS, Du H, Bukowski R, Vogelzang N and Bro WP: Development and validation of a scale to measure disease-related symptoms of kidney cancer. Value Health 10 285-293, 2007.

26. Motzer RJ, Sharma P, McDermott DF, George S, Hammers HJ, Srinivas S, Tykodi SS, Sosman JA, Procopio G, Plimack ER, et al: CheckMate 025 phase iII trial: Outcomes by key baseline factors and prior therapy for nivolumab (nIVO) versus everolimus (eVE) in advanced renal cell carcinoma (rCC). J Clin Oncol 34 (Suppl 2S): Abstr 498, 2016.

27. Thompson RH, Gillett MD, Cheville JC, Lohse CM, Dong H, Webster WS, Krejci KG, Lobo JR, Sengupta S, Chen L, et al: Costimulatory B7-H1 in renal cell carcinoma patients: Indicator of tumor aggressiveness and potential therapeutic target. Proc Natl Acad Sci USA 101: 17174-17179, 2004.

28. Omori S, Kenmotsu H, Abe M, Watanabe R, Sugino T, Wakuda K Ono A, Taira T, Naito T, Murakami H, et al: Changes in pD-11 expression in non-small cell lung cancer by immunohistochemical analysis. J Clin Oncol 33: Abstr e22118, 2015.

29. Gibney GT, Aziz SA, Camp RL, Conrad P, Schwartz BE, Chen CR, Kelly WK and Kluger HM: c-Met is a prognostic marker and potential therapeutic target in clear cell renal cell carcinoma. Ann Oncol 24: 343-349, 2013.

30. Rankin EB, Fuh KC, Castellini L, Viswanathan K, Finger EC Diep AN, LaGory EL, Kariolis MS, Chan A, Lindgren D, et al: Direct regulation of GAS6/AXL signaling by HIF promotes renal metastasis through SRC and MET. Proc Natl Acad Sci USA 111: $13373-13378,2014$.
31. Viola D, Cappagli V and Elisei R: Cabozantinib (XL184) for the treatment of locally advanced or metastatic progressive medullary thyroid cancer. Future Oncol 9: 1083-1092, 2013.

32. Armstrong AJ, Broderick S, Eisen T, Stadler WM, Jones RJ, Garcia JA, Vaishampayan UN, Picus J, Hawkins RE, Hainsworth JD, et al: Final clinical results of a randomized phase II international trial of everolimus vs. sunitinib in patients with metastatic non-clear cell renal cell carcinoma (ASPEN). J Clin Oncol 33: Abstr 4507, 2015.

33. Heng DY, Mackenzie MJ, Vaishampayan UN, Bjarnason GA, Knox JJ, Tan MH, Wood L, Wang Y, Kollmannsberger C, North S, et al: Primary anti-vascular endothelial growth factor (VEGF)-refractory metastatic renal cell carcinoma: Clinical characteristics, risk factors, and subsequent therapy. Ann Oncol 23: 1549-1555, 2012.

34. Hales RK, Banchereau J, Ribas A, Tarhini AA, Weber JS, Fox BA and Drake CG: Assessing oncologic benefit in clinical trials of immunotherapy agents. Ann Oncol 21: 1944-1951, 2010.

35. Wolchok JD, Hoos A, O'Day S, Weber JS, Hamid O, Lebbé C, Maio M, Binder M, Bohnsack O, Nichol G, et al: Guidelines for the evaluation of immune therapy activity in solid tumors: Immune-related response criteria. Clin Cancer Res 15: 7412-7420, 2009

36. Scher HI, Halabi S, Tannock I, Morris M, Sternberg CN, Carducci MA, Eisenberger MA, Higano C, Bubley GJ, Dreicer R, et al; Prostate Cancer Clinical Trials Working Group: Design and end points of clinical trials for patients with progressive prostate cancer and castrate levels of testosterone: Recommendations of the Prostate Cancer Clinical Trials Working Group. J Clin Oncol 26: 1148-1159, 2008.

37. Corsello SM, Barnabei A, Marchetti P, De Vecchis L, Salvatori R and Torino F: Endocrine side effects induced by immune checkpoint inhibitors. J Clin Endocrinol Metab 98: 1361-1375, 2013.

38. Motzer RJ, Rini BI, McDermott DF, Redman BG, Kuzel TM, Harrison MR, Vaishampayan UN, Drabkin HA, George S, Logan TF, et al: Nivolumab for metastatic renal cell carcinoma: Results of a randomized phase iI trial. J Clin Oncol 33: 1430-1437, 2015.

39. Eichelberg C, Vervenne WL, De Santis M, et al: SWITCH: A randomised, sequential, open-label study to evaluate the efficacy and safety of sorafenib-sunitinib versus sunitinib-sorafenib in the treatment of metastatic renal cell cancer. Eur Urol 68 : 837-847, 2015.

40. Motzer RJ, Escudier B, Oudard S, Hutson TE, Porta C, Bracarda S, Grünwald V, Thompson JA, Figlin RA, Hollaender N, et al; RECORD-1 study group: Phase 3 trial of everolimus for metastatic renal cell carcinoma: Final results and analysis of prognostic factors. Cancer 116: 4256-4265, 2010 . 